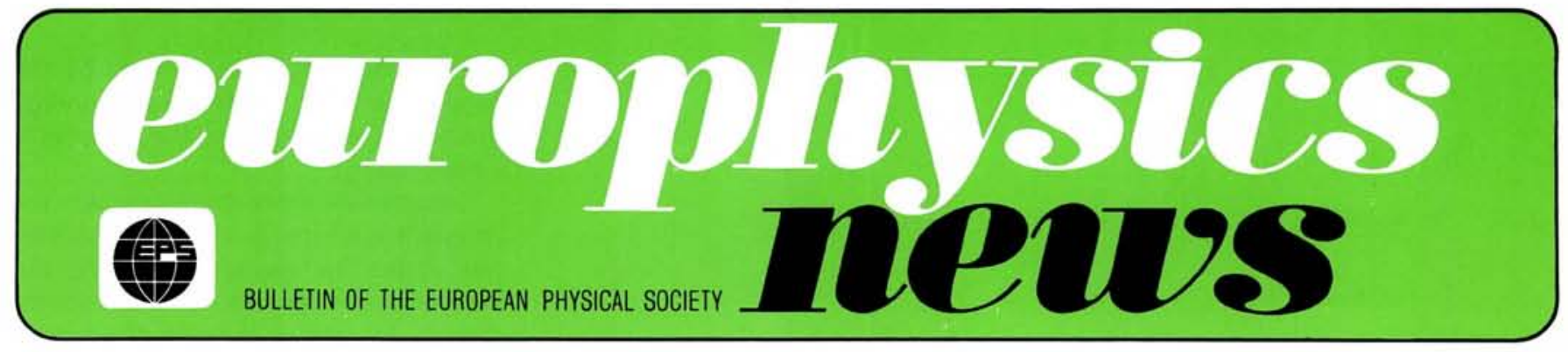

J.A.

Volume 19

Number 4

April 1988

\title{
Magnetic Metals
}

\section{A.R. Mackintosh, Copenhagen}

(Nordita and the University of Copenhagen)

By definition, magnetic metals contain electrons which can transport an electric current, and electrons which provide a net magnetic moment. The fundamental problem which for several decades has exercised those who have been concerned with understanding metallic magnetism is; are they the same electrons? To express it rather more precisely, one might ask whether the conduction electrons in a magnetically ordered metal, for example a ferromagnet, are also responsible for the ordered moment, or whether this is predominantly due to a distinct set of electrons. In the form in which the question is usually asked today: "Are the magnetic electrons localized or itinerant?".

The origin of magnetic ordering in the solid state has been understood in principle since the work of Heisenberg and Dirac. The exchange correlation of electrons of parallel spin holds them apart and hence reduces their Coulomb energy. Since this energy reduction does not occur if the spins are antiparallel, a pair of electrons will tend to align their spins. This coupling may be described as a Heisenberg exchange interaction, proportional to the scalar product of the spin vectors, and falling off rapidly with distance as the overlap of the electron wavefunctions declines. The alignment of the spins is opposed by the resulting increase in, for example, the kinetic energy and, at non-zero temperature, the entropy of disorder. The details of how the Heisenberg and other magnetic interactions are expressed in practice may be very complex, and elaborate experimental and theoretical studies are required for their elucidation.
Diffraction and inelastic scattering of neutrons have played a vital role in allowing the determination of magnetic structures, excitations and interactions. Perhaps the most significant contribution to distinguishing between localized and itinerant magnetic electrons has, however, been made by calculations of the electronic structure and their verification by experimental studies of the Fermi surface. Of particular importance has been the de Haas-van Alphen effect, the oscillatory diamagnetism of metals at low temperatures, which is incomparably the most powerful experimental technique for studying the conduction electrons. From the measurements on $\mathrm{Ni}$ in the early 1960's to the recent results on heavy-fermion systems, such investigations have furnished decisive clues to understanding the ground states of magnetic metals. The excited states of localized magnetic electrons, as manifested for instance in the rare earth metals, are also well understood, but the excitations of itinerant-electron magnets still present an unresolved challenge.

\section{Itinerant Magnetism - \\ Transition Metals}

It has been possible to make realistic calculations of the electronic structures of magnetic metals since the work of Slater in the 1930's, but the subject was given new impetus by the development of density-functional theory by Kohn and others, and linear methods for solving the Schrödinger equation by Andersen. A local single-particle potential for an electron in a lattice may be constructed from the electron density by a prescription which involves assumptions and approximations but no arbitrary parameters, and includes in an average sense the correlation and exchange interactions between the electrons. This potential may be used for self-consistent calculations of the ground-state properties of the electronic system but not, without further elaboration, the excited states. The linear methods are physically transparent and computationally very efficient, so that self-consistent calculations can be performed for complex structures with many atoms per unit cell. The essentials of the band structure may thus be expressed in terms of a small number of parameters, including the energies $C_{\mathrm{nl}}$ of the centres of the bands derived from the various atomic states, and the associated electronic band masses $\mu_{\mathrm{nl}}$, to which the width of the corresponding band is inversely proportional. These parameters may in turn be related to atomic energies and wavefunctions.

Magnetic ordering is associated with atomic states which overlap weakly in the metals: $3 d$ in the transition metals,

$\begin{array}{ll}\text { Contents } & \\ \text { Magnetic Metals } & 41 \\ \begin{array}{l}\text { Very Long Baseline } \\ \text { Interferometry }\end{array} & 46 \\ \begin{array}{l}\text { Chaos in Josephson Junctions } \\ \text { and SQUIDS }\end{array} & 53 \\ \text { Anniversary Seminar } & 55 \\ \text { New EPS Members } & 56 \\ \text { EPS Elections } & 56\end{array}$




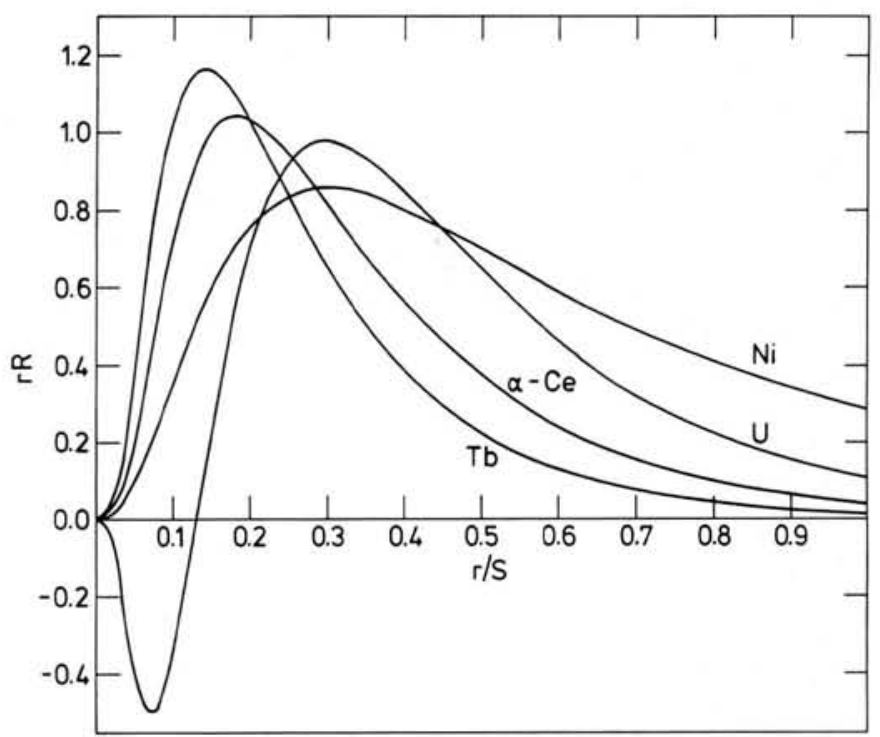

Fig. 1 - Atomic wavefunctions for some magnetic and nearly-magnetic metals, calculated by Herman and Skillman. The $3 d$ wavefunction is shown for $\mathrm{Ni}$, the $4 f$ for $\alpha-\mathrm{Ce}$ and $\mathrm{Tb}$, and the $5 f$ for $U$. The radius is scaled by the Wigner-Seitz radius $S$, so that the amplitude at the value unity indicates the overlap between states on neighbouring atoms.

$4 f$ in the rare earths and $5 f$ in the actinides. In Fig. 1 are shown radial atomic wavefunctions for such states, as a function of the radius scaled with $S$, the radius of the "Wigner-Seitz sphere", which encompasses one atom in the elemental metal. The width of the corresponding band is roughly proportional of the square of the amplitude of the wavefunction on the Wigner-Seitz sphere, which reflects the degree of overlap of states at different sites in the crystal. The associated band masses are: $\mu_{3 \mathrm{~d}}=12$ for $\mathrm{Ni}, \mu_{4 \mathrm{f}}=44$ for $\alpha$-Ce and $\mu_{5 f}=14$ for $\mathrm{U}$, implying much narrower bands than those of the nearlyfree electron gas for which $\mu_{\mathrm{n} 1}=1$.

Whether band theory is applicable when the masses are so high has been a subject of controversy for several decades. In the early 1960's the matter was settled for the $3 \mathrm{~d}$ transition metals when Fawcett and others determined the Fermi surface of $\mathrm{Ni}$ and demonstrated that the $3 \mathrm{~d}$ electrons are itinerant and hence participate in the conduction of electricity on the same footing as, for instance, the 4 s electrons. In such circumstances, the Stoner theory is applicable. The essential assumption is that, owing to the exchange interactions, the centres of the bands corresponding to different spins are split by an amount which is proportional to the magnetization $m$, so that for $\mathrm{Ni}$

$$
C_{3 d, l}-C_{3 d, t}=m l
$$

where the average exchange interaction $I$ can readily be calculated. Assuming that the magnetization is exclusively associated with the $3 \mathrm{~d}$ electrons, which is a good approximation for $\mathrm{Ni}$, the Pauli spin susceptibility of the conduction the $4 \mathrm{~s}$ electrons, is determined by the balance between the decrease in energy due to the exchange and the increase in kinetic energy.

Density-functional theory permits the calculation of the ground-state properties of the $3 \mathrm{~d}$ magnets, including the magnetic moment, the Fermi surface for the two spin states, the crystal structure and the cohesive properties, and the results are generally in excellent agreement with experiment. For example the striking behaviour of $\mathrm{Fe}$ which, upon the application of pressure, changes from a bcc structure with a large moment to a non-magnetic hcp structure, is quantitatively explained by such calculations. Even the magnetic properties of $\mathrm{Cr}$, where the ordered moment varies sinusoidally through the lattice in an antiferromagnetic spin-density-wave structure, may be understood in terms of the energy bands. On the other hand, the excited states are not satisfactorily treated in the simplest form of the band model, in which the elementary magnetic excitations are formed by the promotion of an electron from a filled state to an empty state of opposite spin. The observed low-lying excitations are however spin waves, which may be considered as linear combinations of these Stoner excitations, formed as a result of correlation effects which are neglected in the independent particle model. Although these effects can be included in a relati-
Fig. 2. The degree of band splitting which is much greater for the $3 d$ than electrons is enhanced by this interaction to $\quad \chi_{\mathrm{P}}=\mu_{\mathrm{B}}^{2} N\left(\varepsilon_{\mathrm{F}}\right) /\left(1-N\left(\varepsilon_{\mathrm{F}}\right) /\right)$ where $N\left(\varepsilon_{\mathrm{F}}\right)$ is the number of electron states per unit energy range (the density) at the Fermi level. From this relation it is clear that, if $N\left(\varepsilon_{F}\right) /$ exceeds ferromagnetic state with a spontaneous spin polarization results, as illustrated in

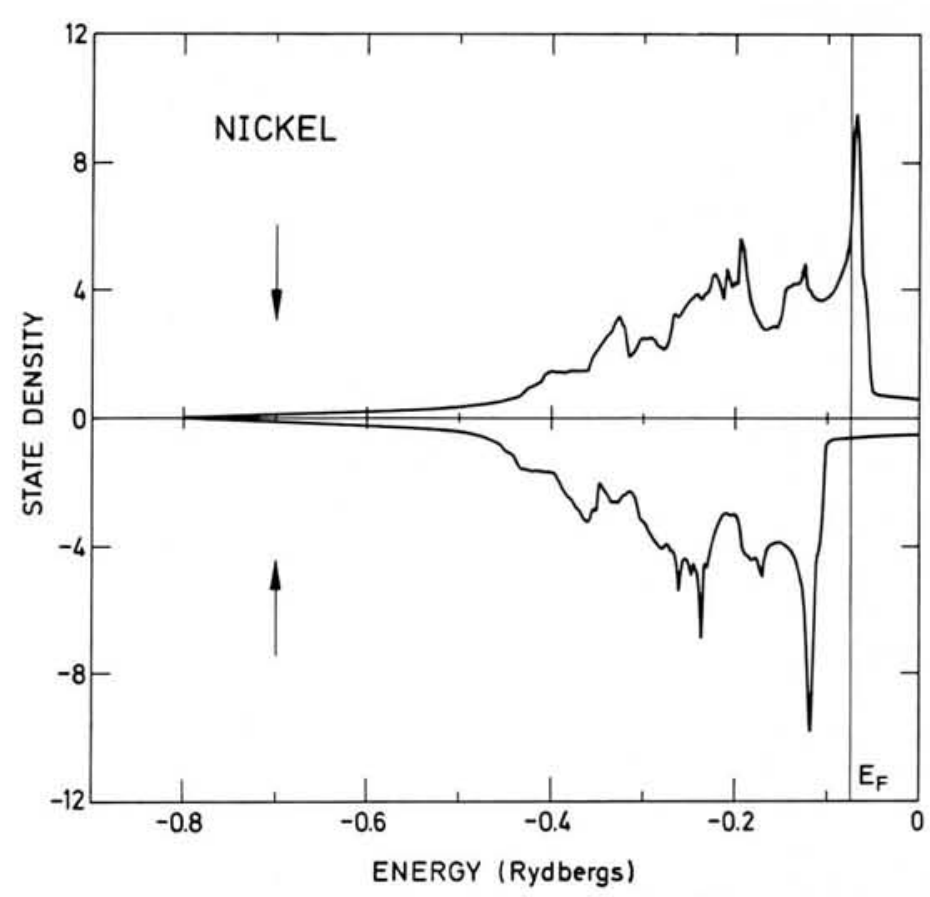

Fig. 2 - The number of electron states per unit energy for the different spin states in ferromagnetic Ni, calculated by Skriver. The bottoms of the $4 \mathrm{~s}$ bands for up and down spin almost coincide at $-0.77 R y$ but the narrow $d$ bands, which give rise to the large state density near the Fermi level, are self-consistently shifted relative to each other by the exchange. The top of the spin-upd band is at about - 0.1 Ry below the Fermi level, and the band is therefore filled. The spin-down $d$ band however contains about 0.6 holes per atom, and the difference gives rise to the ferromagnetic moment in $\mathrm{Ni}$. 


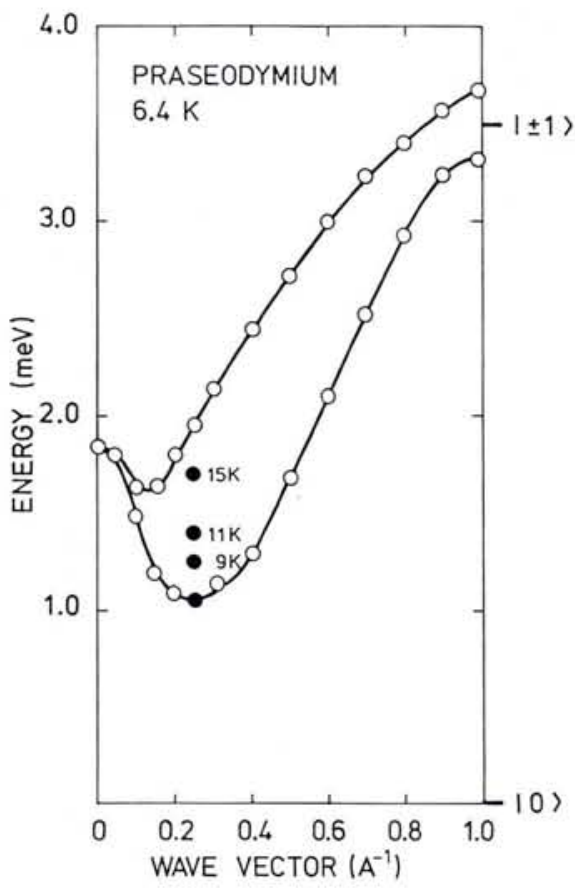

Fig. 3 - The effect of the magnetic interactions in the rare earth metal Pr. In the atomic ground state $J=5$, and the crystalline electric field in the metal splits the $(2 J+1)-d e-$ generate $\left|M_{J}\right\rangle$ states, the lowest two of which are shown. The indirect exchange interaction couples these states on different ions, giving rise to a collective excitation whose energy depends upon the wavelength. The $\mid \pm 1>$ doublet is split by the anisotropy of the exchange. As indicated, the mode of lowest energy is strongly temperature dependent, and the hyperfine coupling to the nuclear moments gives an antiferromagnetic structure below about 50 millikelvins, whose periodicity matches the wavelength of this mode.

vely straightforward manner, and the spin-wave dispersion relations thereby calculated, the temperature dependence of the magnetization and the Curie temperature still present problems. Furthermore the $3 d$ electron energies measured by photoemission differ quantitatively from the calculated band structure, so that the exchange splitting in $\mathrm{Ni}$, for example, is calculated to be too large, and localized "atomic" excitations, not present in the band picture, are observed experimentally.

\section{Localized Moments - \\ Rare Earth Metals}

The rare earth series, through which the $4 f$ levels are successively filled, provide the opposite extreme to the $3 \mathrm{~d}$ transition metals. The $4 \mathrm{f}$ charge clouds overlap so little, and are so well shielded from their surroundings by the 5 s and 5 p electrons, that the most appropriate starting point (apart from $\mathrm{Ce}$, which we consider later) for determining their magnetic properties is to consider them as localized states subject to the same intra-atomic interactions as in the free stom, and hence with a ground state and total angular momentum $\mathbf{J}$ determined by Hund's rules. The $5 d, 6 s$ and $6 p$ states constitute the conduction bands, whose two spin states are split in the magnetically ordered phases by the interaction with the localized $4 \mathrm{f}$ moments, rather than by the self-exchange which determines the band splitting in the magnetic transition metals. The Fermi surfaces corresponding to these two spin states have been studied by Young and others, and their results are in good agreement with the predictions of band-structure calculations.

The magnetic properties of the rare earth metals are determined by the interactions of the local moments with their surroundings. The two-ion coupling between them is predominantly due to the indirect exchange mechanism, in which a conduction electron interacts successively by Heisenberg exchange with the spin component of the local moments on different sites, and thereby couples them together. The anisotropic crystalline electric field arising from the inhomogeneous surroundings also act on the ions, and the long range of the exchange interaction and the strong crystal-field anisotropy combine to produce exotic magnetic structures, first studied in detail by Koehler, Legvold and their colleagues. Of particular interest are those structures, such as the helix, in which the magnetic and lattice periodicities are incommensurable, thus breaking the translational symmetry. Such structures have proved to be of importance in a number of areas.
In contrast to the itinerant-electron magnets, the magnetic interactions in the localized rare earth systems cannot yet be predicted satisfactorily from first principles. However if they can be inferred, for instance from neutron experiments, both the magnetic ground state and excitations, and hence a wide range of magnetic properties, may be accurately calculated. The static magnetic properties are conveniently determined from a mean-field theory, in which the splitting of the $2 J+1$ levels (corresponding to the different $M_{j}$ values) of a particular moment by the crystal fields and exchange is calculated by assuming that the surrounding moments have their thermally-averaged values. The excitations may be deduced from a timedependent extension of this method known as the random phase approximation. The experimental study of these excitations through inelastic neutron scattering, particularly by Bjerrum Møller and his colleagues, has provided a detailed picture of the very rich magnetic behaviour of the rare earths, as well as a precise measurement of the magnetic interactions. The interplay between these is illustrated in Fig. 3 .

\section{f-band Metals}

An intriguing exception to this picture of the rare earths is provided by $\mathrm{Ce}$. At room temperature it crystallizes in a fcc structure, with one $4 \mathrm{f}$ electron which is believed to behave much like the localized $4 \mathrm{f}$ states in the heavier metals. When the temperature is reduced below about $100 \mathrm{~K}$ however, the structure collapses, while retaining its fcc symmetry, to

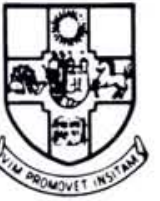 \\ UNIVERSITY OF BRISTOL \\ CHAIR IN PHYSICS}

The University invites applications for a Chair in Physics, following the retirement of Professor J.F. Nye, FRS. It is expected that the person appointed will be an experimental condensed-matter physicist.

Suitably qualified candidates are invited to submit applications, including the names of three referees, not later than 29th April, 1988. Further particulars of the appointment may be obtained from the Registrar and Secretary, University of Bristol, Senate House, Bristol. BS8 1TH. England. 


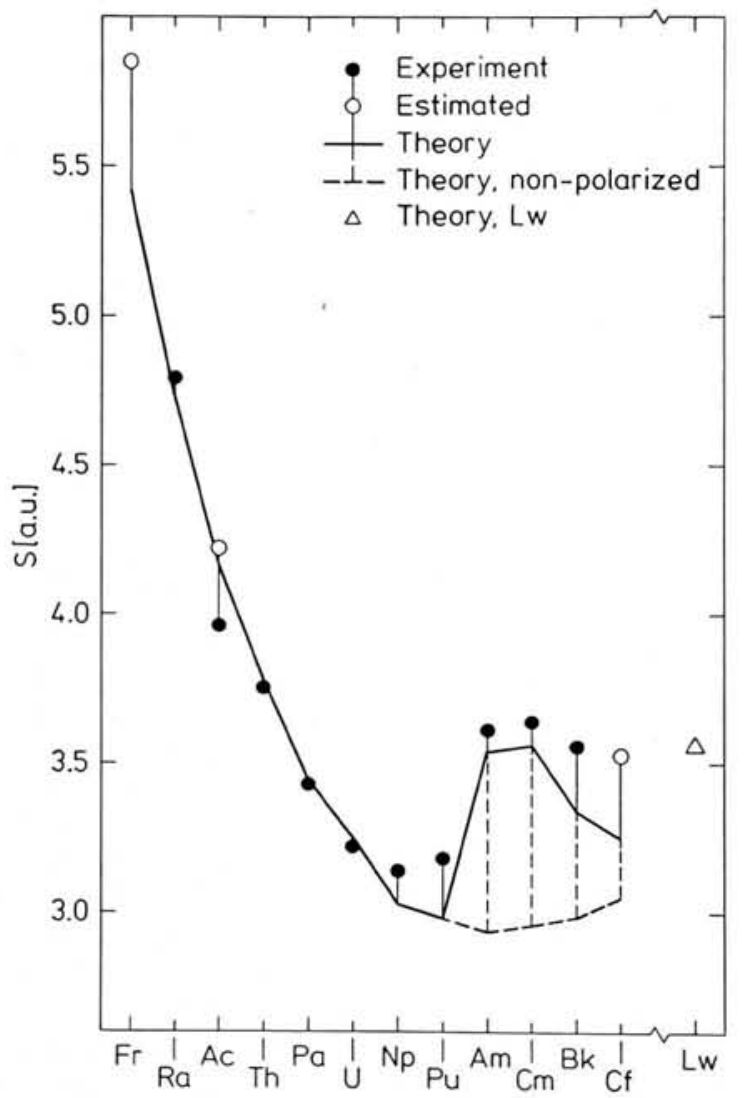

Fig. 4 - The Wigner-Seitz radius $S$ (proportional to the lattice constants) in the actinide metals. In the non-magnetic light actinides the $5 f$ band makes a large contribution to the cohesion, causing a rapid contraction with atomic number reminiscent of the behaviour of the transition metals. At Am however the f electrons become localized and thereafter make little contribution to the total energy. The heavy actinides are therefore magnetic and behave like the rare earths, with atomic volumes which vary little with atomic number. This behaviour is well accounted for by the calculations of Skriver, Johansson and Andersen, who find that the magnetic state is indeed stable beyond Am.

roughly $80 \%$ of the original volume. There is very convincing evidence that this $\gamma-\alpha$ transition is due to the delocalization of the $4 \mathrm{f}$ electrons which then form a band, as first proposed by Gustafson and his collaborators. As may be seen from Fig. 1, the overlap of the $4 f$ wavefunctions in the low-temperature allotrope $\alpha-\mathrm{Ce}$, though not large, is much greater than in the heavy rare earths and the total $4 \mathrm{f}$ band width is over $1 \mathrm{eV}$, so that the $4 \mathrm{f}$ electrons make a substantial contribution to the cohesive energy. The excited states of the $4 \mathrm{f}$ electrons, measured for example by photoemission, show characteristics of both localized and itinerant states. $\alpha-\mathrm{Ce}$ is paramagnetic and, as discovered by Wittig, even becomes superconducting at high pressures. Since it is extremely difficult to prepare in the form of pure single crystals, detailed experimental measurements of the electronic structure have not been performed, but Crabtree and co-workers have studied the Fermi surface in the related compound $\mathrm{CeSn}_{3}$ and shown that the $4 \mathrm{f}$ electrons are itinerant and contribute to the conduction bands. The measured Fermi surface agrees well with that deduced from band calculations but observed masses are about a factor five higher than the calculated band masses. We shall return to these large mass enhancements.

A clear manifestation of the localization of $f$ electrons occurs in the actinide metals. As may be seen in Fig. 1, the overlap of the $5 \mathrm{f}$ electrons in the light actinides is intermediate between the transition and rare earth metals. Consequently they form bands and, since the cohesion of metals is due to the electron gas, they make an essential contribution to the cohesive properties; the complex and unsymmetrical structures of the light actinides are primarily due to the angular dependence of the $f$ wavefunctions. In the heavy actinides, starting with $A m$, the $f$ electrons are however localized, because of the contraction of the wavefunction with increasing atomic number, and therefore make little contribution to the cohesive energy. The heavy actinides thus behave similarly to the rare earths, with localized moments and symmetrical structures whose atomic volume varies slowly with atomic number. This behaviour is faithfully reproduced by the band calculations of Skriver and his colleagues. As shown in Fig. 4, the atomic volume at first decreases rapidly with atomic number, reflecting the increasing $5 f$ binding, but increases abruptly at the localization transition in Am. In the band calculations, this localization is manifested as the appearance of a spontaneous magnetic polarization. When such a polarization has developed, band theory is no longer sufficient, since it does not adequately take account of, for example, the coupling between the orbital moments. Indeed the applicability of band theory to the description of localized states is still an open question. A periodic array of localized states can, in principle, formally be described in terms of filled bands, but correlation effects may be so strong that the currently used local approximation to density-functional theory cannot be applied to the ground state, and in any case the excited states are not generally related in any simple way to the calculated energy bands.

Itinerant $f$ electrons are at the focus of interest in magnetism research at present. In different circumstances, depending essentially on the bandwidth, f-band metals display such disparate features as magnetic ordering, superconductivity, mixed valence and heavyfermion behaviour. The last of these phenomena occurs in such compounds as $\mathrm{CeCu}_{6}$ or $\mathrm{UPt}_{3}$, in which the direct $f-f$ overlap is negligible, but a very narrow f-band, with a large band mass, is formed by overlap with states on the surrounding atoms. This large mass is further enhanced by spin fluctuations. When a metal is close to spontaneous magnetic polarization, an itinerant electron is accompanied by a cloud of spin fluctuations which increase the effective mass, just as the lattice distortion around a moving electron gives rise to the phonon enhancement of the mass. The phenomenon of spin-fluctuation enhancement has been recognized since the 1960's, when it was realized that the d electrons in Pd, which is almost ferromagnetic, should have a larger effective mass than band-structure calculations would predict. Whereas phonons give an enhancement of typically $30 \%$ however, and spin fluctuations in Pd, which are predominantly of long-wavelength, are even less effective, the strong fluctuations which occur throughout the Brillouin zone in f-band systems may give as much as a factor 20 . The result is an electronic heat capacity, which is proportional to the mass, which may be a thousand times greater than in ordinary metals.

Apart from this large enhancement of the effective mass, the Fermi surface experiments of Taillefer, Lonzarich and their collaborators on $\mathrm{UPt}_{3}$ are in good accord with several independent band calculations. This compound is also superconducting, as are $\mathrm{UBe}_{13}$ and $\mathrm{CeCu}_{2} \mathrm{Si}_{2}$, the latter being the first heavy-fermion superconductor, discovered by Steglich and his colleagues in 1979. It has been proposed that the attractive interaction between the electrons which is required to stabilize the superconducting state is caused by antiferromagnetic spin fluctuations in 
these materials, rather than the conventional phonon mechanism, but this question, which may be related to the mechanism for high-temperature superconductivity in conducting oxides, is by no means finally settled. Similarly, speculations that the Cooper pairs may be more complex than the usual singlet pairs of states of opposite spin have been neither confirmed nor definitively disproved.

The excited states of heavy-fermion systems are also incompletely understood. The overlap of the $f$ electrons with the surrounding wavefunctions is so light that thermal fluctuations can break the coherence of the itinerant state at temperatures as low as $10-100$ $\mathrm{K}$. As a result, they behave at higher temperatures as an assembly of independent localized $f$ states and the properties of such systems vary rapidly and, at present, unpredictably with decreasing temperature as the coherent state is being established.

\section{Conclusion}

As we have seen, the question posed at the beginning - "Are the magnetic electrons localized or itinerant?" - has different answers, depending upon the circumstances. The magnetism of the $3 \mathrm{~d}$ transition metals is due to band elec- trons which contribute to the Fermi surface and the electrical conductivity. The $4 f$ electrons in the rare earths, on the other hand, are localized with energies lying well below the Fermi level. In the $f$-band metals, the electrons which display actual or incipient magnetism are itinerant at low temperatures but tend to become localized as the temperature is increased.

In illustrating these features, we have mostly considered the metallic elements, but current research in magnetism is primarily concerned with magnetic compounds, in which the magnetic properties of the different atomic species are generally very different, so that the magnetism may be very inhomogeneously distributed. An extreme example is the magnetic superconductors, in which one set of electrons is magnetic and another is superconducting. The high-temperature oxide superconductors illustrate this behaviour. The $4 \mathrm{f}$ electrons on the rare earth ions may order antiferromagnetically with only a very slight influence on the superconductivity. In the superconducting heavy-fermion compounds, on the other hand, the situation appears to be more complex. Although the superconductivity of $\mathrm{UPt}_{3}$, for example, may be largely due to the $\mathrm{Pt}$ atoms, heat capacity and neutron

\section{The Foundation for Fundamental Research on Matter}

promotes fundamental scientific research on matter in the Netherlands generally and at universities in particular. The Foundation tries to achieve this purpose by stimulating research in new fields of physics, by co-ordinating existing research projects and by involving its institutes and working groups in the education and training of young physicists.

The Foundation has an opening for two

\section{Postdoctoral Positions}

at the Department of Nuclear Physics at the University of Utrecht (The Netherlands).

The experimental nuclear physics programme concentrates on electron scattering and heavy-ion physics. Heavy-ion research is focussed on the dynamics of intermediate energy reactions and on the behaviour of highly excited nuclei, whereas the electron experiments emphasize the dynamical aspects of neutron emission processes. The experiments are performed at the accelerators of NIKHEF-K (Amsterdam) and KVI (Groningen) and at facilities abroad (SARA, Grenoble, SATURNE, Saclay). The group is also actively involved in the development of complex detection systems that will be commissioned at the new Dutch facilities (the $800 \mathrm{MeV} \mathbf{1 0 0 \%}$ duty factor electron stretcher ring at Amsterdam and the superconducting cyclotron AGOR at Groningen, both now under construction).

The candidate is expected to participate actively in the current experimental programme and to provide new directions for future research.

The duration of the positions is for a maximum of three years. The positions are available from mid 1988.

Applications to be sent to Personnel Department, Laboratory of Experimental Physics, University of Utrecht, P. O. Box 80.000, NL-3508 TA Utrecht, The Netherlands.

For further information contact: Prof. Dr. P.K.A. de Witt Huberts (for the electron scattering position): Tel.: (20) 5922145 / 5922143 ; and Dr. R. Kamermans (for the heavy-ion position): Tel. (30) 532517 / 531492 .

\section{WANTED}

Complete collection of the following journals, years 1980-1986:

Surf. Sci., Appl. Surf. Sci., Surf. Sci. Reports; J. Phys. F; Appl. Phys. A (Springer); J. Vac. Sci. Technol. A+B.

Fair prices paid.

J. Osterwalder, Phys. Dept.,

University of Fribourg,

CH - 1700 FRIBOURG, Switzerland

diffraction studies indicate that the $5 f$ electrons on the $U$ sites are involved in the superconducting state, even though recent measurements by Aeppli, Broholm and their colleagues show that they also carry a small ordered moment.

There remain many unexplained mysteries associated with heavy-fermion systems, and the understanding of the interplay between heavy-fermion behaviour, magnetism and superconductivity is perhaps the major goal of current magnetism research, with the enticing prospect of helping to elucidate the origins of high-temperature superconductivity. The progenitor of the hightemperature superconductors, $\mathrm{LaCuO}_{4}$, exhibits antiferromagnetism, even though band calculations indicate that the exchange interaction is far too small to induce magnetic ordering in a pure, perfect crystal. Furthermore, strong magnetic fluctuations persist well above the magnetic transition temperature. These observations have intensified speculation that magnetism and superconductivity are intimately linked in the high-temperature superconductors.

\section{FURTHER READING}

\section{Transition Metals}

Lonzarich G.G. in Electrons at the Fermi Surface, Ed. M. Springford (Cambridge University Press) 1980.

Andersen O.K., Jepsen O. and Glötzel D. in Highlights of Condensed-Matter Theory (Italian Physical Society, Bologna) 1985.

Rare Earth Metals

Mackintosh A.R., Physics Today 30 (1977) 23.

Legvold S., in Ferromagnetic Materials, Vol. 1, Ed. E.P. Wohlfarth (North-Holland, Amsterdam) 1980.

Actinides

Brooks M.S.S., Johansson B. and Skriver H.L., in Handbook on the Physics and Chemistry of the Actinides, Eds. A.J. Freeman and G.H. Lander (North-Holland, Amsterdam) 1984.

Heavy-Fermion Systems

Stewart G.R., Rev. Mod. Phys. 56 (1984) 755.

Fisk Z., Ott H.R., Rice T.M. and Smith J.L., Nature 320 (1986) 124. 